\title{
Breather Stability in One Dimensional Lattices with a Symmetric Morse Potential
}

H. CORTEZ, E. DRIGO FILHO, J.R.RUGGIERO, Departamento de Física, IBILCE, UNESP, 15054-000 São José do Rio Preto, SP, Brasil.

\begin{abstract}
Harmonic one dimensional lattice with an additional Morse potential on site has been used to describe DNA macromolecules properties. We analyze a modification of this lattice introducing a symmetric Morse potential. The existence and stability of the breather is studied in this modified system. We obtain harmonic bifurcation and determine the effective mass of the mobile breather.
\end{abstract}

Keywords. Anti-continuous limit, Symmetric Morse potential, Breather, Center of energy.

\section{Introduction}

Physical aspects of the DNA has received large attention from the scientific community (see, for example, ref. [1] for an experimental overview on the theme), in particular, several theoretical models have been introduced to study thermodynamics [2] and dynamics [3-5] properties of this macromolecules. In the end of the 1980 decade Peyrard and Bishop (PB) introduced a mathematical model to analyze the DNA. In the PB model, DNA molecule is mimicked by two parallel strands composed by chains of masses (the nucleotides) coupled elastically one to another. The strands are coupled by a Morse potential simulating the H-bond between the bases pair in the macromolecule. The Hamiltonian for the Peyrard-Bishop model [2] is given by :

$$
H=H_{p}+H_{V}
$$

where

$$
H_{p}=\sum_{j}\left\{\frac{p_{u_{j}}^{2}}{2 m}+\frac{p_{v_{j}}^{2}}{2 m}\right\}
$$

represents the kinetic energy of the system, and

$$
H_{V}=\sum_{j}\left\{\frac{K}{2}\left[\left(u_{j}-u_{j-1}\right)^{2}+\left(v_{j}-v_{j-1}\right)^{2}\right]\right\}+\sum_{j} V\left(u_{j}-v_{j}\right)
$$

\footnotetext{
${ }^{1}$ hernan@ibilce.unesp.br

2 elso@ibilce.unesp.br

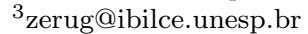


is the potential energy. In the equation (1.2), $p_{u_{j}}$ and $p_{v_{j}}$ are the linear moment. For simplicity we consider that the mass $m$ and the constants $\mathrm{K}$ are equals for all nucleotides.

Introducing a new set of coordinates:

$$
y_{j}=\frac{u_{j}-v_{j}}{\sqrt{2}}, \quad x_{j}=\frac{u_{j}+v_{j}}{\sqrt{2}}
$$

we get a new form to the Hamiltonian

$H=\sum_{j}\left\{\frac{p_{x_{j}}^{2}}{2 m}+\frac{p_{y_{j}}^{2}}{2 m}\right\}+\sum_{j}\left\{\frac{K}{2}\left[\left(x_{j}-x_{j-1}\right)^{2}+\left(y_{j}-y_{j-1}\right)^{2}\right]\right\}+\sum_{j} V\left(\sqrt{2} y_{j}\right)$

As usual in the DNA analysis [2,3] our interest is to study the optical Hamiltonian of this latter equation given by:

$$
H=\sum_{j}\left\{\frac{p_{y_{j}}^{2}}{2 m}\right\}+\sum_{j}\left\{\frac{K}{2}\left[\left(y_{j}-y_{j-1}\right)^{2}\right]\right\}+\sum_{j} V\left(\sqrt{2} y_{j}\right) .
$$

The equations of motion for (1.6) are the system of discrete nonlinear KleinGordon $(\mathrm{KG})$ equations $(\mathrm{j}=1,2, \ldots, \mathrm{N})$

$$
y_{j}^{\prime \prime}+K\left(2 y_{j}-y_{j-1}-y_{j+1}\right)+V^{\prime}\left(\sqrt{2} y_{j}\right)=0
$$

with V' being the derivative of the potential. Breathers are periodic and localized solutions of (1.7) [6]. The dynamic of the PB model is obtained from eq. (1.7) explored in reference [2] and our results in this case are similar to that reference. In this model the function $V\left(u_{j}-v_{j}\right)$ is given by a Morse potential.

The main results of this work is concerning the symmetric Peyrard-Bishop (SPB) model, where the potential $V\left(u_{j}-v_{j}\right)$ is symmetric (it is defined in the next section). We also observe the existence of harmonic bifurcation and we determine initial conditions to move the breather.

\subsection{The model and the formulation of the problem}

In the original PB model it is used the Morse potential in eq. (1.5), i.e.,

$$
V\left(y_{i}\right)=D\left[e^{-a\left(y_{i}\right)}-1\right]^{2} .
$$

In the present work, we modify the original model by using a symmetric Morse potential (SPB model) given by

$$
V\left(y_{i}\right)=D\left[e^{-a\left|y_{i}\right|}-1\right]^{2},
$$


in eq. (1.5). The constants $a$ and $\mathrm{D}$ are specific parameters related with the width and deep of the potential, respectively.

The acoustic Hamiltonian describes a chain of harmonically coupled oscillators and do not deserve any new physical knowledge. Then, in what follow, we will be treating only the equations of motion derived from the optical Hamiltonian. Considering, for simplicity, $\mathrm{m}=1, \mathrm{D}=1 / 2$ and $\mathrm{a}=1$, the equation of motion for the studied models are given by equation (1.7) and we are interested in their time reversible and periodic solutions. Breather [3], with a given frequency $w_{b}$ and period $\mathrm{T}=2 \pi / w_{b}$, can be obtained from nonlinear discrete systems of the form (1.7). MacKay and Aubry proved analytically their existence [7]. In the present work, numerical methods have been used to obtain breathers using truncated Fourier series for the coordinates $y_{n}(t)$ :

$$
y_{n}=z_{n}{ }^{0}+\sum_{k=0}^{k_{m}} 2 z_{n}{ }^{k} \cos \left(k w_{b} t\right)
$$

Substituting this expression for $y_{n}(t)$ in (1.7) we obtain a system of algebraic equations given by:

$$
k^{2} w_{b}{ }^{2} z_{n}{ }^{k}+K\left(2 z_{n}{ }^{k}-z_{n-1}{ }^{k}-z_{n+1}{ }^{k}\right)+V_{n}{ }^{k}=0
$$

which depend on the control parameter $\mathrm{K}$, and $V_{n}{ }^{\prime k}$ is the Fourier coefficient for the periodic function $V^{\prime}\left(y_{n}(t)\right)$ The dynamics of the DNA model was originally studied with soliton techniques [2]. However, in this work we use the anti-continuous limit method [7] to get the solution. The first step of this method is to solve numerically the system of equations (1.7) in the uncoupled limit, $\mathrm{K}=0$. Using the above truncated Fourier series with $w_{b}=0.8$ and $k_{m}=17$ represents the eighteen coefficients of (1.10) which are obtained by usual numerical calculation with MATLAB [8]. The next step to create the breather is to introduce the coupling between the neighborhood oscillators, i. e., to solve the dynamical equations with $K \neq 0$, using the previous isolated oscillator solution as a trial function. The numerical results are obtained for $\mathrm{N}=21$ (as the number of oscillators in $(1.10)$ ), $w_{b}=0.8$, we start from a trial solution at $K=0$ and increasing the parameter $K$ by small steps. Each obtained solution for a value of $\mathrm{K}$ is used as the trial solution for the new value of this parameter, replaced by $K+\delta$, in a modified Newton process. At the beginning, the breather ("bright breather") solution is obtained considering only one oscillator vibrating with equal amplitude from their equilibrium position. Figure 1 show a example of such solution. The Fourier coefficients were obtained by the Newton's method [8]. The solutions for a short period of time are presented in Figure 1. In this figure, the oscillators are numbered from -10 to +10 with the central one labeled with $\mathrm{n}=0(\mathrm{~N}=21)$.

The stability of the breather solution can be performed numerically. The goal is to get the behavior of the system around the periodic solutions $u_{n}(t)$. Let us introduce a new function $U_{n}(t)=y_{n}(t)+\epsilon_{n}(t)$, where $y_{n}(t)$ is the periodic breather solution of the SPB model shown in Figure 1 and $\epsilon_{n}(t)$ is a perturbation. Imposing 


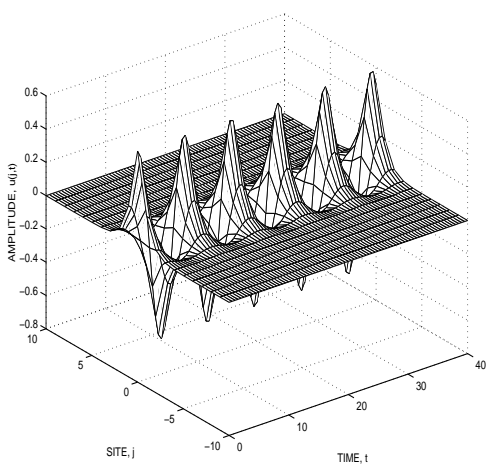

Figura 1: Spatial breather configuration of the SPB model with the control parameters $\mathrm{K}=0.1297$ and $w_{b}=0.8$.

that $U_{n}(t)$ must satisfy the system (1.7) and expanding around $y_{n}(t)$ to first order (linearization), we obtain the following system of equations for $\epsilon_{j}(t)$.

$$
\epsilon_{j}{ }^{\prime \prime}+k\left(2 \epsilon_{j}-\epsilon_{j-1}-\epsilon_{j+1}\right)+V^{\prime \prime}\left(\sqrt{2}\left(y_{j}(t)\right) \epsilon_{j}=0\right.
$$

The Floquet theory [9] is used for differential equations with periodic coefficients. In our cases (symmetric and non-symmetric Morse potential), $y_{n}$ are periodic functions and the system (1.12) can be transformed in Hill's equations with periodic coefficients since $V^{\prime \prime}\left(\sqrt{2}\left(y_{j}(t)\right)\right.$ is also a continuous periodic function. The linear stability of these solutions can be obtained from the eigenvalues of the Floquet matrix F0 , called Floquet multipliers. The Floquet matrix F0 is given by the fundamental matrix $\Phi(t)$ associated to the system (1.12) at the period T. The equations (1.12) can be transformed in a system of first order equations by introducing

$$
\xi^{\prime}=\pi, \pi^{\prime}=-V^{\prime \prime}(y(t)) \xi-K J \xi
$$

where $\xi$ and $\pi$ are $\mathrm{N}$ dimensional vectors, represented by column matrix of dimension $\mathrm{N} \times 1$. $\left[\mathrm{V}^{\prime \prime}\left(y_{n}(\mathrm{t})\right)\right]$ and $[\mathrm{J}]$ are $\mathrm{NxN}$ matrix with elements given by $\left[V^{\prime \prime}\left(y_{n}(t)\right)\right]_{i, j}=\mathrm{V} "\left(y_{n}(t)\right) \delta_{i, j}$ and $[J]_{i, j}=2 \delta_{i, j}-\delta_{i, j-1}-\delta_{i, j+1}$, respectively, $\delta_{i, j}$ being the Kronecker delta symbol. The appropriate periodic boundary conditions used in the calculation is given by $y_{0}=y_{N}$ and $y_{1}=y_{N+1}$.

\section{Floquet multipliers}

In short, we can construct a vector $\Omega(t)=(\xi(t), \pi(t))$ and a periodic matrix [A] $(2 \mathrm{~N}$ x $2 \mathrm{~N})$ composed by four blocks with dimension $\mathrm{N}$ x $\mathrm{N}$ so that $\Omega(t)$ satisfies the equation

$$
\Omega^{\prime}=A(t) \Omega .
$$


The fundamental matrix $\Phi(t)$ can be construct from the $2 \mathrm{~N}$ linearly independent solution of (1.13) corresponding to different initial conditions, defined as a column vector with $2 \mathrm{~N}-1$ zeros and 1 in the $\mathrm{i}$-th position $(\mathrm{i}=1, \ldots, 2 \mathrm{~N})$ such that, for any instant t, $\Phi(t)$ satisfy

$$
\Phi^{\prime}=A(t) \Phi
$$

The equation (2.1) is a Floquet type equation. The matrix $[\mathrm{A}]$ is periodic, i.e., $[\mathrm{A}(\mathrm{t}+\mathrm{T})]=[\mathrm{A}(\mathrm{t})]$. Then, it is easy to show that $\Phi(t+T)$ is also solution of (1.13) with each element being a linear combination of all elements of $\Phi(t)$. Thus, it can be written as

$$
\Phi(t+T)=\Phi(t) M
$$

where $\mathrm{M}$ is a matrix of constant elements. Obviously, by construction $\Phi(0)$ is the identity matrix and $\Phi(T)=\mathrm{M}$ is known as the monodromy matrix. In this case, $\mathrm{M}$ is the Floquet matrix F0.

The eigenvalues of monodromy matrix are the Floquet multipliers. As discussed in reference [9] the solution is stable (bounded) if the modules of Floquet multipliers are equal to one, and unstable if at least one multiplier has modulus different from one. In this last case, the perturbation solution $\epsilon_{n}(t)$ will increase in time because after p periods of time, the equation (2.3) leaves us to

$$
\Phi(t+p T)=\Phi(t) M^{p}
$$

that will blow up if at least one of the multiplier has modulus greater than 1 . To ensure linear stability, all eigenvalues $f_{\nu}=e^{i \theta_{\nu}}$ of M have to lie on the unit circle in a complex plane, i.e., the Floquet arguments $\theta_{\nu}$ must be real-valued. We can construct a breather mobile using a harmonic bifurcation [3]. The intuitive analogy with the pendulum is helpful, because in this case we have two possibilities. We are interested here in rotating solutions above the separatrix, a kinetic perturbation must be exerted [3].

The center of energy for the system is given by [3]

$$
X_{E}=\frac{\sum_{n} n H_{n}}{H}
$$

where the density energy $H_{n}$ is the form

$$
H_{n}=\left\{\frac{p_{y_{n}}^{2}}{2 m}\right\}+\left\{\frac{k}{4}\left[\left(y_{n}-y_{n-1}\right)^{2}+\left(y_{n+1}-y_{n}\right)^{2}\right]\right\}+V\left(\sqrt{2} y_{n}\right) .
$$

We can use the profiles of the stationary breather for these parameters and the velocity is a vector which the components are given by [3]

$$
v_{n}(0)=\left(y_{n+1}(0)-y_{n-1}(0)\right) / 2
$$


We can define a perturbation velocity in terms of the parameter $\lambda$. The components of this vector perturbation $\mathrm{V}$ are given by

$$
V_{n}(0)=\lambda\left(v_{n}(0) / v\right)
$$

where $v$ is the norm of the vector of the components $v_{n}(0)$.

This velocity can be used as a small perturbation added to the initial conditions. Thus, the kinetic energy added to the breather by the initial 'kick' is $(1 / 2) \lambda^{2}$. The effective mass can be defined through the relation

$$
m^{*} V_{b}^{2} / 2=\lambda^{2} / 2
$$

where $V_{b}$ is the breather velocity of the center of energy obtained by the equations (1.7).

\section{Stability and mobility for SPB model}

The second derivative of the symmetric Morse potential (1.9) is given by

$$
V^{\prime \prime}=2 \exp \left(-2\left|y_{n}\right|\right)-\exp \left(-\left|y_{n}\right|\right)
$$

and should be used in the equations of motion (1.12).

Solving this system of equations, we observe breather stability for many values of the coupling parameter $\mathrm{K}(0<K<0.28)$ and frequency $w_{b}=0.8$. This bifurcation is characterized when two real eigenvalues of Floquet multipliers are different from one for $\theta=0$, as showed in figure $2 \mathrm{a}$. In this figure we note that two eigenvalues are out of the unit circle and this exact solution is linearly unstable. For the coupling $\mathrm{K}=0.28$ and $w_{b}=0.8$ there is a harmonic bifurcation ( fig. 2(a)). In this case we can construct a mobile breather following the methodology of the reference [3], described in the preceding section, using a perturbative parameter $\lambda$.

We determine for the parameters $\mathrm{K}=0.28, w_{b}=0.8, \lambda=0.1$ and $\mathrm{v}=3 / 800$ (fig. 2 (b)) the effective mass. It is, in this case, $m=(\lambda / v)^{2}=711.11$. When we use $\mathrm{K}=0.35$ the effective mass is 493.8 .

The breather motion can be characterized by the perturbation parameter $(\lambda)$. In the SPB model the critical value is $\lambda=0.1$. We note that the mobility of the breather depends on the proximity of control parameter $\mathrm{K}$ from the region of harmonic bifurcation (figure 2(a)). For $\mathrm{K}=0.28, w_{b}=0.8, \lambda=0.01$ the breather remains pinned because the center of energy $X_{E}$ remains fixed (figure 2(b)). On the other hand, for $\mathrm{K}=0.28, w_{b}=0.8$ and $\lambda=0.1$ the center of energy is displaced to others sites of the lattice, i.e. we get mobile breather. Finally for $\lambda=1$ the velocity of the center of energy is not uniform.

\section{Conclusions and Discussion}

From the analysis of SPB model, we could shown the existence and the stability of the breathers. In particular, the behavior of the mobile breathers is determined in 


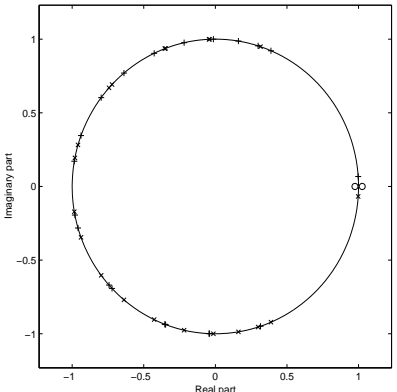

(a)

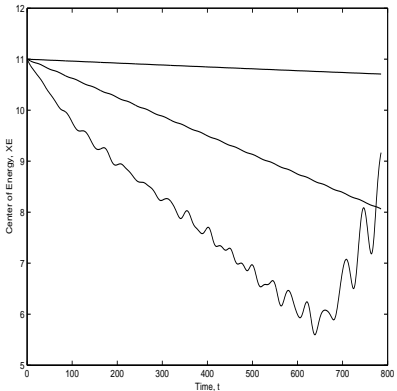

(b)

Figura 2: Distribution of the Floquet eigenvalues of the instability matrix of the breather and the Center of energy. (a)Distribution on the unit circle of the extended Floquet matrix in the SPB model for $w_{b}=0.8$ and $\mathrm{k}=0.28$. Two eigenvalues are out of the unit circle and this exact solution is linearly unstable. (b)Center of energy $\left(X_{E}\right)$ as a function of the critical parameter with $\mathrm{K}=0.28$ and $w_{b}=0.8$, the parameter $\lambda$ are $\lambda=0.01$ (upper line), $\lambda=0.1$ (middle line) and $\lambda=1$ (bottom line)

terms of the center of energy. This behavior depends on the initial conditions, the parameter $\mathrm{K}$ and $w_{b}$. We obtain for the SPB model the breather stability for the parameters $0<K<0.28$ and $w_{b}=0.8$, while for the same value of the frequency the original $\mathrm{PB}$ model gives stable breathers for $\mathrm{K}$ in the range $0<K<0.13$ [3]. We observe that the range of the stability in terms of the parameter $\mathrm{K}$ is approximately the double for the symmetric case. The result indicates that the SPB model is more stable than the $\mathrm{PB}$ one. We also determine that the amplitudes of the breathers for the PB model are larger than the amplitudes obtained for the SPB model.

In terms of the effective mass, we observe that it decrease if the couple parameter $\mathrm{K}$ increase. This behavior is also observed for the PB model [3].

Finally, we would like to express that the center of energy is useful in the analysis of the interaction of breathers and impurities. Then, the results obtained here can be used to study this kind of lattices which is more realistic for physical application, for instance, in DNA macromolecule [4]. On the other hand, the existence of the mobile breather can be useful to understand the localized 'hot spot', i.e. a local accumulation of energy, which might be pinned in the lattice or may travel through it [10] and the existence of thermodynamic phase transitions [11].

\section{Acknowledgment}

The authors would like to thank J. Cuevas , M. Tsuchida and W. Cortez for useful discussions. This work was partially supported by CNPq and FAPESP.

Resumo. Redes não lineares com o potencial de Morse tem sido usados para descrever as propriedades do DNA. Analizamos uma modificação desta rede usando o potencial de Morse simétrico. A existência e a estabilidade dos breathers são estudadas neste sistema. Os resultados mostram a existência de bifurcação harmônica e a massa efetiva do breather móvel também foi determinada. 


\section{Referências}

[1] W. Saenger, "Principles of Nucleotidic Acid Structure", Spring Verlag, New York, 1984.

[2] M. Peyrard, "Statistical Mechanics of a Nonlinear model for DNA", Physica Review Lett. ,Vol. 62, pp. 2755-2758, 1989.

[3] J. Cuevas, "Localización y Transferencia de Energia en Redes Anarmónicas No Homogéneas", Ph. D. Thesis, Universidad de Sevilla, Sevilla, España, 2003.

[4] K. Forinash, "Interaction of discrete breathers with impurity modes", Physica Review E ,Vol. 49, pp.3400-3411, 1994.

[5] P. Augusto, E.Drigo,J.Ruggiero, "Statistical Model to DNA melting", Ecl. Quim.S.P., Vol. 26, pp.77-85, 2001.

[6] S. Flach, C.R. Willis, "Discrete breathers", Physics Reports ,Vol. 295, pp.181$264,1998$.

[7] R.S.Mackay, S. Aubry , "Proof of existence of breathers for time-reversible for Hamiltonian networks of weakly coupled oscillators ", Nonlinearity Vol. 7, pp. 1623-1643, 1994.

[8] The Student Edition of MATLAB, "User's guide", Prentice Hall, Massachusetts, 2004.

[9] F.C. Hoppensteadt, "Analysis and Simulation of Chaotic Systems", SpringVerlag, New York, 2000.

[10] G.P. Tsironis and S.Aubry , "Slow relaxation Phenomena induced by Breathers in nonlinear lattices", Physica Review Letters ,Vol. 77, pp. 5225-5228, 1996. ..

[11] N. Theodorakopoulos, "Thermodynamic instabilities in one-dimensional lattices: a finite-size scaling approach", Physica Review E ,Vol. 68, pp. (026109)1-4, 2003. 